\title{
Fluorinated graphene oxide for enhanced S and X-band microwave absorption
}

\author{
P. M. Sudeep, ${ }^{1,2}$ S. Vinayasree,,${ }^{1,3}$ P. Mohanan, ${ }^{4}$ P. M. Ajayan, ${ }^{5}$ T. N. Narayanan, ${ }^{2}$ \\ and M. R. Anantharaman ${ }^{1, a)}$ \\ ${ }^{1}$ Department of Physics, Cochin University of Science and Technology, Kochi 682022, Kerala, India \\ ${ }^{2}$ TIFR-Centre for Interdisciplinary Sciences, Tata Institute of Fundamental Research, \\ Hyderabad 500075, India \\ ${ }^{3}$ Government Polytechnic College, Thirurangadi, Velimukku P.O., Malappuram 676317, Kerala, India \\ ${ }^{4}$ Department of Electronics, Cochin University of Science and Technology, Kochi 682022, Kerala, India \\ ${ }^{5}$ Department of Materials Science and NanoEngineering, Rice University, Houston, Texas 77005, USA
}

(Received 10 January 2015; accepted 26 May 2015; published online 3 June 2015)

\begin{abstract}
Here we report the microwave absorbing properties of three graphene derivatives, namely, graphene oxide (GO), fluorinated GO (FGO, containing 5.6 at. \% Fluorine (F)), and highly FGO (HFGO, containing 23 at. \% F). FGO is known to be exhibiting improved electrochemical and electronic properties when compared to GO. Fluorination modifies the dielectric properties of GO and hence thought of as a good microwave absorber. The dielectric permittivities of GO, FGO, and HFGO were estimated in the $\mathrm{S}(2 \mathrm{GHz}$ to $4 \mathrm{GHz})$ and $\mathrm{X}(8 \mathrm{GHz}$ to $12 \mathrm{GHz})$ bands by employing cavity perturbation technique. For this, suspensions containing GO/FGO/HFGO were made in NMethyl Pyrrolidone (NMP) and were subjected to cavity perturbation. The reflection loss was then estimated and it was found that $-37 \mathrm{~dB}$ (at $3.2 \mathrm{GHz}$ with $6.5 \mathrm{~mm}$ thickness) and $-31 \mathrm{~dB}$ (at $2.8 \mathrm{GHz}$ with $6 \mathrm{~mm}$ thickness) in the $\mathrm{S}$ band and a reflection loss of $-18 \mathrm{~dB}$ (at $8.4 \mathrm{GHz}$ with $2.5 \mathrm{~mm}$ thickness) and $-10 \mathrm{~dB}$ (at $11 \mathrm{GHz}$ with $2 \mathrm{~mm}$ thickness) in the $\mathrm{X}$ band were achieved for $0.01 \mathrm{wt}$ \% of FGO and HFGO in NMP, respectively, suggesting that these materials can serve as efficient microwave absorbers even at low concentrations. (C) 2015 AIP Publishing LLC.
\end{abstract}

[http://dx.doi.org/10.1063/1.4922209]

There is an increasing demand for wide band microwave absorbing materials so as to prevent electromagnetic radiations emanating from most of the modern electronic gadgets, viz., computers, mobiles, etc. ${ }^{1}$ Newer materials having good absorption characteristics are the need of the hour in order to reduce electromagnetic interference (EMI) in circuits, chips, and radiation controllers. ${ }^{2}$ Because of the ever-growing requirement for microwave absorbers with strong absorption ability in the wideband combined with light weight and thinness, new materials with superlative absorption characteristics are scouted for. ${ }^{3,4}$ Carbon based materials are important candidates for microwave absorption eventhough they offer low impedance matching. Composites containing carbon black and carbon nanotubes (CNTs) along with magnetic materials are increasingly being employed as microwave absorbers. ${ }^{5}$ Recently, two dimensional layered materials and their composites were investigated for their microwave absorbing properties. $^{6-9}$ Graphene and their derivatives are also seen as potential microwave absorbers due to their superior electronic, thermal, and mechanical properties. One of the major advantages of graphene based systems is that they possess large surface area. It is also reported that layered structured materials serve as efficient microwave absorbers rather than rod or tube shaped materials. ${ }^{10}$

Graphene oxide (GO) is a non-conductive hydrophilic carbon material with hydroxyl, epoxy, carboxylic functional

\footnotetext{
${ }^{\text {a) }}$ Author to whom correspondence should be addressed. Electronic mail: mraiyer@yahoo.com. Tel.: +91-484-2577404.ext. 26.
}

groups on its planes and edges. ${ }^{11}$ They can be reduced under high temperature or using strong reducing agents to form conducting reduced GO (RGO). Thus, the addition or removal of functionalities enables tuning of the properties of GO so as to suit them for a variety of applications. ${ }^{11-16}$ Incorporation of various elements in the GO backbone offers various possibilities to alter their properties which will be useful in energy storage, hydrophobic coating, and thermal management among others. ${ }^{17,18}$ Recently, GO based materials were investigated for their microwave absorbing properties. ${ }^{19-22}$ However, most of these materials are composites consisting of GO and ferrites or GO and appropriate magnetic nanoparticles. ${ }^{20,23-26}$ R-GO and their composites with magnetic components exhibit enhanced microwave absorption due to RGO's conducting nature and are being extensively studied by many research groups. ${ }^{20,24,27}$

Fluorinated GO (FGO) attracted the attention of several researchers among other derivatives of GO. Recently, the authors reported enhanced thermal conductivity for FGO nanofluids ${ }^{28}$ and also found that fluorinated graphene (powder) can be used as cathodes for primary batteries with enhanced performance. ${ }^{29}$ Romero-Aburto et al. found FGO as a good magnetic resonance imaging (MRI) contrast enhancing agent. ${ }^{30}$ It is presumed that on fluorination of GO, the $\mathrm{C}-\mathrm{F}$ bond imparts excellent oxidative and thermal stability. Moreover, fluorine being electronegative, the $\mathrm{C}-\mathrm{F}$ bond gives rise to high polarity. Absorption mainly comes from the dielectric loss because of polarization and the defects present in fluorinated carbon systems. ${ }^{20}$ 
To investigate the effect of fluorination of GO on the microwave absorbing properties, we synthesized GO, FGO ( $\mathrm{F} \sim 5.6$ at. \%), and highly FGO (HFGO, F $\sim 23$ at. \%, calculated using X-ray photoelectron spectroscopy (XPS) shown in supplementary Figure S1). ${ }^{40}$ The $S$ and $X$ band regions were chosen as it assumes exceptional significance in the electromagnetic spectrum as it can find expedient applications in different areas such as in radars, satellites, direct broadcast satellites, mobile services, and also in WiMAX technology. ${ }^{31-33}$ GO, FGO, and HFGO were prepared by using an "improved method" reported elsewhere. ${ }^{34}$ The details of synthesis, chemical structure, properties, and detailed experimental procedures are described in our previous reports. ${ }^{18,28}$ Suspensions containing $0.01 \mathrm{wt}$ \% of GO, FGO, and HFGO were prepared in N-methyl pyrrolidone (NMP) via extensive ultrasonication. Complex dielectric permittivity measurements of GO, FGO, and HFGO suspensions were carried out using a vector network analyzer (Rohde \& Schwaz ZVB4) in the S band, and for X-band measurements the cavity is attached to a four port Agilent network analyzer. For this, suspensions were filled in a capillary tube of known dimensions measured using a traveling microscope and inserted into a rectangular cavity having dimensions $30.8 \times 7.2 \times 3.4 \mathrm{~cm}$. The cavity was perturbed at different transverse electrical modes of $\mathrm{TE}_{102}$ and $\mathrm{TE}_{106}$ in the $\mathrm{S}$ band and $\mathrm{TE}_{103} \mathrm{TE}_{101}$ in the $\mathrm{X}$ band. When a sample is inserted inside the cavity, the resonant frequency and quality factor change and the complex permittivity is calculated using the formula ${ }^{35}$

$$
\begin{gathered}
\varepsilon^{\prime}=\frac{\mathrm{V}_{\mathrm{c}}\left(\mathrm{f}_{\mathrm{c}}-\mathrm{f}_{\mathrm{s}}\right)}{2 \mathrm{~V}_{\mathrm{s}} \mathrm{f}_{\mathrm{s}}}+1, \\
\varepsilon^{\prime \prime}=\frac{\mathrm{V}_{\mathrm{c}}}{4 \mathrm{~V}_{\mathrm{s}}}\left(\frac{1}{\mathrm{Q}_{\mathrm{s}}}-\frac{1}{\mathrm{Q}_{\mathrm{c}}}\right),
\end{gathered}
$$

where $\mathrm{V}_{\mathrm{s}}$ and $\mathrm{V}_{\mathrm{c}}$ are the volume of the material and cavity, respectively, $\mathrm{f}_{\mathrm{s}}$ and $\mathrm{f}_{\mathrm{c}}$ are the resonance frequencies with and without the suspension, and $\mathrm{Q}_{\mathrm{s}}$ and $\mathrm{Q}_{\mathrm{c}}$ are the corresponding quality factors of the sample and cavity, respectively, and are given by

$$
Q_{s}=\frac{f_{s}}{f_{c}-f_{s}}, \quad Q_{c}=\frac{f_{c}}{f_{c}-f_{s}} .
$$

Figure 1 depicts the variation of complex dielectric permittivity with frequency. The real part of dielectric permittivity is found to be 13 and 32 for GO and HFGO, respectively. There is a large increase in the dielectric permittivity of FGO when compared to GO and HFGO. It may be noted that FGO exhibited an enhanced permittivity $\left(\varepsilon^{\prime}\right)$ of 63 at $2.55 \mathrm{GHz}$, while the real part of permittivity decreased from 63 to 61 for FGO over the $\mathrm{S}$ band frequency region of 2.55 to $3.6 \mathrm{GHz}$. FGO also exhibited an increase in dielectric loss with an increase from 4.5 to 6.4 compared to GO.

The variation of complex dielectric permittivity in the $\mathrm{X}$ band from $7.6 \mathrm{GHz}$ to $12 \mathrm{GHz}$ is shown in Figure 2. Here too the permittivity of FGO is high when compared to GO or HFGO. The imaginary part of permittivity is also a bit higher in the case of FGO which increased from 6 to 22 compared to $\mathrm{GO}$ at $8.25 \mathrm{GHz}$. It is found that in both $\mathrm{S}$ and $\mathrm{X}$ bands, the permittivity is higher for FGO when compared to GO and HFGO. This is due to the lattice defects created by fluorination of graphene oxide. Fluorine can induce charge separation $^{30}$ in the FGO lattice and further these defects act as polarization centers. It is also known that the interaction of fluorine atom with GO layers is via covalent bonding ${ }^{26}$ and the $\mathrm{sp}^{2}$ to $\mathrm{sp}^{3}$ ratio of carbon atoms is higher in the case of FGO compared to HFGO but similar to GO. ${ }^{36}$ Even with similar $\mathrm{sp}^{2}$ to $\mathrm{sp}^{3}$ ratio, FGO possesses high dielectric permittivity which in turn shows the role of fluorine in the enhancement. The difference in bandgap between these two types of carbon atoms $\left(\mathrm{sp}^{2}\right.$ and $\left.\mathrm{sp}^{3}\right)$ creates band fluctuation in GO layers which acts as defects in the electronic band. Moreover, the induced polarisation between fluorine and carbon could be contributing to the dielectric loss of FGO.

The other possible reason for the decrease in the permittivity of HFGO is the decrease in the electronic conductivity of fluorinated GO upon increasing the F content. Many researchers have reported that fluorination of graphite, CNT, or graphene alters their electronic conductivity. ${ }^{37,38}$ In the case of HFGO, the high amount of fluorination makes it more insulating which in turn will affect the dielectric permittivity of HFGO.

The microwave absorption characteristics of a material are analyzed on the basis of its complex dielectric permittivity and magnetic permeability. Good absorbers should have low reflection coefficient and good absorption coefficient. The electromagnetic reflection property of a material is typically illustrated in terms of the reflected power of a plane wave from a metal terminated absorber system.
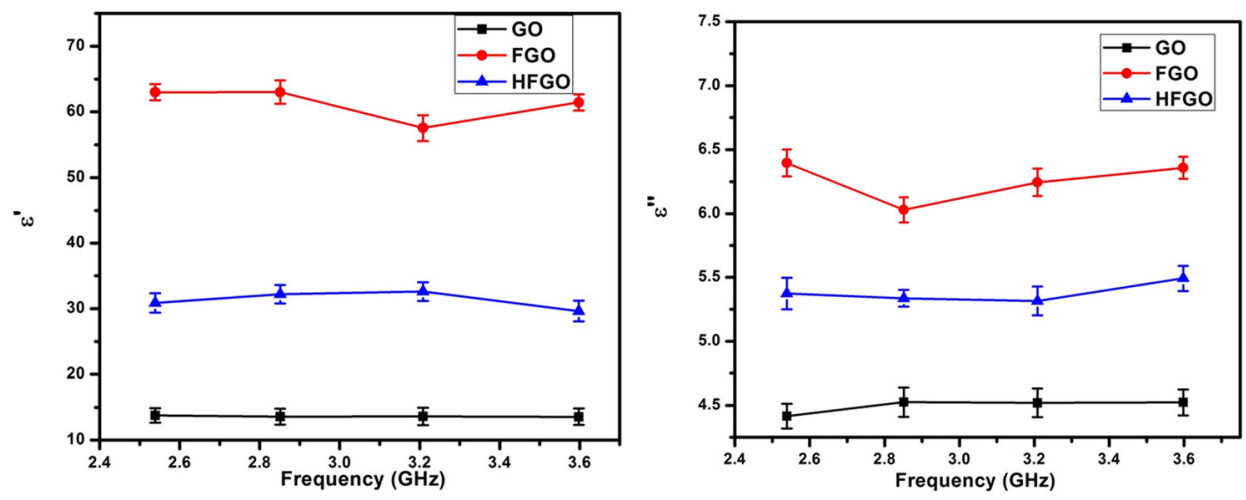

FIG. 1. Variation of complex dielectric permittivity of GO, RGO, and FGO with frequency in the $\mathrm{S}$ band. 

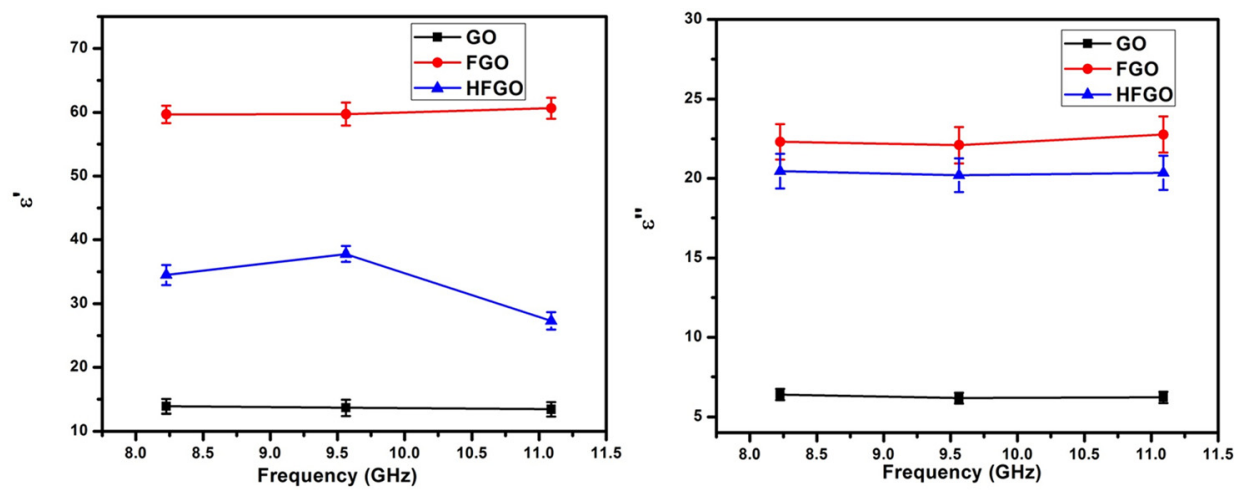

FIG. 2. Variation of complex dielectric permittivity of GO, FGO, and HFGO with frequency in the $\mathrm{X}$ band.

Electromagnetic waves entering a material are absorbed effectively if there is good impedance matching between the materials. Here, the reflection loss was estimated by using the surface impedance modelling. The reflectivity of the material is expressed as ${ }^{26}$

$$
\mathrm{R}=20 \log _{10}[\Gamma] \mathrm{dB}
$$

where $\left.\Gamma=\left(Z_{\text {in }}-Z_{0}\right) / Z_{\text {in }}+Z_{0}\right) ; Z_{\text {in }}$ is the input impedance of wave absorber and $Z_{0}$ is that of free space, wherein

$$
\mathrm{Z}_{\mathrm{in}}=\mathrm{Z}_{0} \sqrt{\frac{\mu_{\mathrm{r}}}{\varepsilon_{\mathrm{r}}}} \tanh (\gamma \cdot \mathrm{t}),
$$

where $\gamma=\alpha+\mathrm{i} \beta, \alpha$ is the attenuation constant, and $\beta$ is the phase constant.

Variation in reflection loss of GO, FGO, and HFGO in the $\mathrm{S}$ and $\mathrm{X}$ band has been evaluated and is shown in Figure 3. Reflection losses of $-28.5 \mathrm{~dB},-37 \mathrm{~dB}$, and $-31 \mathrm{~dB}$ have been obtained for thicknesses of $5 \mathrm{~mm}$, $6.5 \mathrm{~mm}$, and $6 \mathrm{~mm}$ for GO, FGO, and HFGO, respectively,
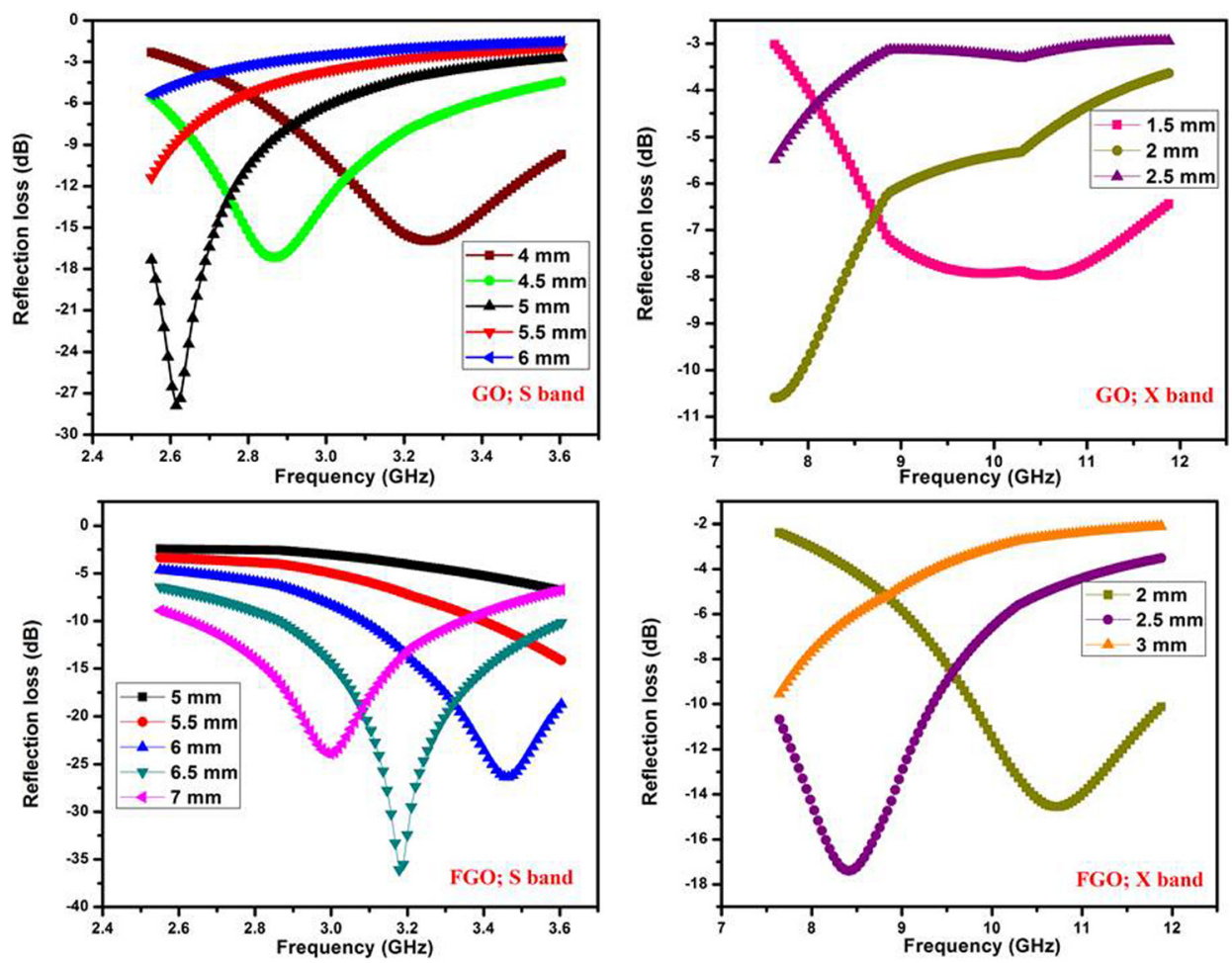

FIG. 3. Variation of reflection loss of $\mathrm{GO}, \mathrm{FGO}$, and HFGO in the $\mathrm{S}$ and $\mathrm{X}$ bands.
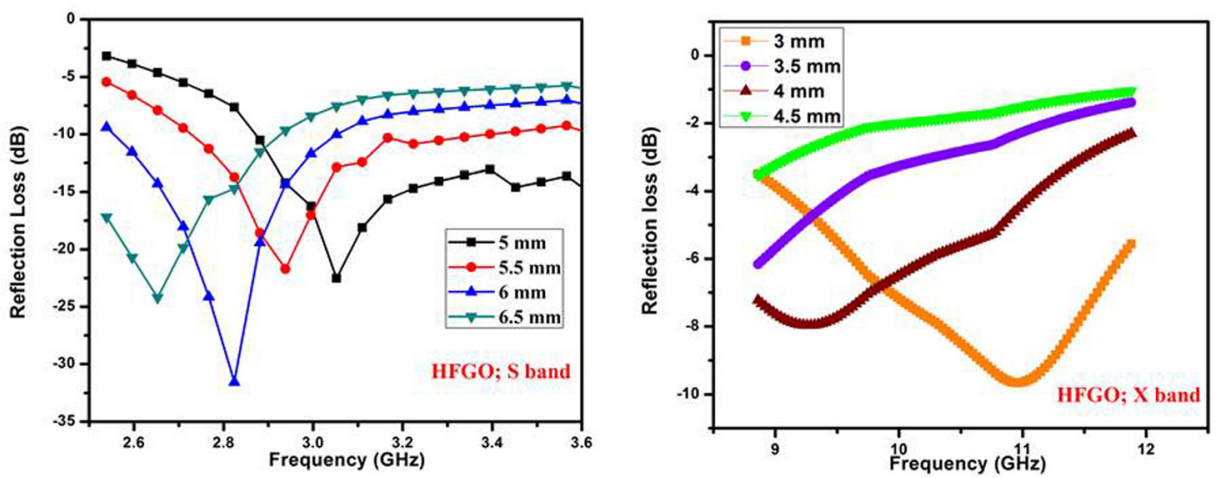
in the $\mathrm{S}$ band. Reflection losses of $-8 \mathrm{~dB},-18 \mathrm{~dB}$, and $-10 \mathrm{~dB}$ corresponding to thicknesses $1.5 \mathrm{~mm}, 2.5 \mathrm{~mm}$, and $3 \mathrm{~mm}$ for GO, FGO, and HFGO, respectively, have been achieved in the $\mathrm{X}$ band frequency region. It should be noted that the fluorination gives absorption with considerable large bandwidth of $180 \mathrm{MHz}$ and $165 \mathrm{MHz}$, respectively, for FGO and HFGO. Furthermore, it is noticed that HFGO exhibits stronger microwave absorption eventhough it possesses lower permittivity than FGO. Many factors could be contributing to the enhanced microwave absorption of fluorinated GO system. The absorption mechanism is attributed mainly to the dielectric loss and coincidentally very high in the case of FGO and HFGO compared to GO. There is a small variation in dielectric loss between FGO and HFGO which indicates that the absorption will be higher and the difference in reflection loss will be lesser which is obvious from Figure 3. Apart from dielectric loss, electronic relaxation also plays a role in absorption. In the case of FGO and HFGO, the effect of fluorination generates lattice defects and C-F clusters can act as polarization centers. So the electromagnetic waves interact with the charge induced by fluorination which in turn produces polarization and attenuate electromagnetic wave ${ }^{20}$ leading to a good absorption. The reflection loss from GO, FGO, and HFGO is briefly compared with the existing reported carbon based materials (see supplementary material ${ }^{40}$ ) and it is found that the GO, FGO, and HFGO can act as efficient microwave shielding materials. Recently, researchers investigated the electron transfer kinetics of FGO and compared with GO and $\mathrm{RGO}^{39}$ Despite the presence of a large number of functional groups in FGO, it is seen that the electron transfer rate is higher than that of RGO (more electronically conductive than FGO). Thus, it can be inferred that the presence of fluorine in the GO matrix not only influences the surface properties of GO but it also alters the density of states near the Fermi level too. Present study reveals that along with electronic properties, $\mathrm{F}$ can also affect the surface polarizations and hence the dielectric relaxation. A detailed investigation is necessary to substantiate these results.

In conclusion, fluorinated graphene oxide layers with different fluorine percentages were studied for their microwave absorption properties. It has been found that fluorination modifies the dielectric permittivity of graphene oxide, and this in turn increases the microwave absorption in the $\mathrm{S}$ and $\mathrm{X}$ bands for thicknesses of $1.5-6.5 \mathrm{~mm}$. Hence, this study opens further avenue for developing radiation shielding paints based on FGO for meeting the everlasting demand for radiation protection in various fields.

P.M.S. acknowledges University Grants Commission (UGC), Government of India for financial assistance under Research Fellowship in Sciences for Meritorious Students (RFSMS) (C2/018450/UGC-PHY-III/12-13). M.R.A acknowledges Department of Science and Technology (DST), Government of India for the financial support through DST-Nanomission project (SR/NM/NS-120/ 2010(G)).
${ }^{1}$ F. Qin and C. Brosseau, J. Appl. Phys. 111, 061301 (2012).

${ }^{2}$ E. Ma, J. Li, N. Zhao, E. Liu, C. He, and C. Shi, Mater. Lett. 91, 209-212 (2013).

${ }^{3}$ J. Zheng, H. Lv, X. Lin, G. Ji, X. Li, and Y. Du, J. Alloys Compd. 589, 174-181 (2014)

${ }^{4}$ R. K. Srivastava, T. N. Narayanan, A. P. R. Mary, M. R. Anantharaman, A. Srivastava, R. Vajtai, and P. M. Ajayan, Appl. Phys. Lett. 99, 113116 (2011).

${ }^{5}$ X. Sun, J. He, G. Li, J. Tang, T. Wang, Y. Guo, and H. Xue, J. Mater. Chem. C 1, 765 (2013).

${ }^{6}$ D. Chen, G. S. Wang, S. He, J. Liu, L. Guo, and M. S. Cao, J. Mater. Chem. A 1, 5996 (2013).

${ }^{7}$ M. Zong, Y. Huang, H. Wu, Y. Zhao, Q. Wang, and X. Sun, Mater. Lett. 114, 52-55 (2014).

${ }^{8}$ P. B. Liu, Y. Huang, and X. Sun, ACS Appl. Mater. Interfaces 5, 12355-12360 (2013).

${ }^{9}$ Y. Chen, G. Xiao, T. Wang, Q. Ouyang, L. Qi, Y. Ma, P. Gao, C. Zhu, M. Cao, and H. Jin, J. Phys. Chem. C 115, 13603-13608 (2011).

${ }^{10}$ Y. Zhan, F. Meng, Y. Lei, R. Zhao, J. Zhong, and X. Liu, Mater. Lett. 65, 1737-1740 (2011).

${ }^{11}$ D. Bhuvaneswaria and N. Kalaiselvi, Dalton Trans. 43, 18097-18103 (2014).

${ }^{12}$ S. Chen, J. Duan, Y. Tang, B. Jin, and S. Zhang, Nano Energy 11, 11-18 (2015).

${ }^{13}$ W.-J. Ong, L.-L. Tan, S.-P. Chai, and S.-T. Yonga, Chem. Commun. 51, 858-861 (2015).

${ }^{14}$ Y.-T. Xu, Y. Guo, C. Li, X.-Y. Zhou, M. C. Tucker, X.-Z. Fu, R. Sun, and C. P. Wong, Nano Energy 11, 38-47 (2015).

${ }^{15}$ L. Fei, Q. Lin, B. Yuan, G. Chen, P. Xie, Y. Li, Y. Xu, S. Deng, S. Smirnov, and H. Luo, ACS Appl. Mater. Interfaces 5(11), 5330-5335 (2013).

${ }^{16}$ Z. Wang and C.-J. Liu, Nano Energy 11, 277-293 (2015).

${ }^{17}$ A. L. M. Reddy, A. Srivastava, S. R. Gowda, H. Gullapalli, M. Dubey, and P. M. Ajayan, ACS Nano 4(11), 6337-6342 (2010).

${ }^{18}$ A. Mathkar, T. N. Narayanan, L. B. Alemany, P. Cox, P. Nguyen, G. H. Gao, P. Chang, R. R. Aburto, S. Mani, and P. M. Ajayan, Part. Part. Syst. Charact. 30(3), 266-272 (2013).

${ }^{19}$ D. Zhang, D. Zhao, J. Zhang, and L. Bai, J. Alloys Compd. 589, 378-383 (2014).

${ }^{20}$ B. Zhang, C. Lu, and H. Li, Mater. Lett. 116, 16-19 (2014).

${ }^{21}$ G. M. Barbosa, M. M. Mosso, C. Vilani, D. R. G. Larrue, E. C. Romani, and F. L. F. Junior, Microwave Opt. Technol. Lett. 56, 560-563 (2014)

${ }^{22}$ H. Yu, T. Wang, B. Wen, M. Lu, Z. Xu, C. Zhu, Y. Chen, X. Xue, C. Sun, and M. Cao, J. Mater. Chem. 22, 21679 (2012).

${ }^{23}$ C. Hu, Z. Mou, G. Lu, N. Chen, Z. Dong, M. Hua, and L. Qu, Phys. Chem. Chem. Phys. 15, 13038 (2013).

${ }^{24}$ V. Sunny, W. Tabis, D. SakthiKumar, Y. Yoshida, P. Mohanan, and M. R. Anantharaman, Mater. Lett. 64(10), 1130-1132 (2010).

${ }^{25}$ S. Vinayasree, M. A. Soloman, V. Sunny, P. Mohanan, P. Kurian, P. A. Joy, and M. R. Anantharaman, J. Appl. Phys. 116, 024902 (2014).

${ }^{26}$ S. Vinayasree, M. A. Soloman, V. Sunny, P. Mohanan, P. Kurian, and M. R. Anantharaman, Compos. Sci. Technol. 82, 69-75 (2013).

${ }^{27}$ M. Zong, Y. Huang, Y. Zhao, X. Sun, C. Qu, D. Luo, and J. Zheng, RSC Adv. 3, 23638 (2013)

${ }^{28}$ P. M. Sudeep, J. Taha-Tijerina, P. M. Ajayan, T. N. Narayanan, and M. R. Anantharaman, RSC Adv. 4, 24887-24892 (2014).

${ }^{29}$ D. Damien, P. M. Sudeep, T. N. Narayanan, M. R. Anantharaman, P. M. Ajayan, and M. M. Shaijumon, RSC Adv. 3, 25702-25706 (2013).

${ }^{30}$ R. Romero-Aburto, T. N. Narayanan, Y. Nagaoka, T. Hasumura, T. M. Mitcham, T. Fukuda, P. J. Cox, R. R. Bouchard, T. Maekawa, D. S. Kumar et al., Adv. Mater. 25, 5632 (2013).

${ }^{31}$ A. Wadhawan, D. Garrett, and J. M. Perez, Appl. Phys. Lett. 83, 2683 (2003).

${ }^{32}$ P. C. P. Watts, W.-K. Hsu, A. Barnes, and B. Chambers, Adv. Mater. 15, 600 (2003).

${ }^{33}$ X. D. Chen, G. Q. Wang, Y. P. Duan, and S. H. Liu, J. Phys. D: Appl. Phys. 40, 1827 (2007).

${ }^{34}$ D. C. Marcano, D. V. Kosynkin, J. M. Berlin, A. Sinitskii, Z. Sun, A. Slesarev, L. B. Alemany, W. Lu, and J. M. Tour, ACS Nano 4, 4806-4814 (2010). 
${ }^{35}$ T. N. Narayanan, V. Sunny, M. M. Shaijumon, P. M. Ajayan, and M. R. Anantharaman, Electrochem. Solid-State Lett. 12(4), K21-K24 (2009).

${ }^{36}$ P. Chantharasupawong, R. Philip, T. N. Narayanan, P. M. Sudeep, A. Mathkar, P. M. Ajayan, and J. Thomas, J. Phys. Chem. C 116, 25955-25961 (2012).

${ }^{37}$ K.-I. Ho, C.-H. Huang, J.-H. Liao, W. Zhang, L.-J. Li, C.-S. Lai, and C.-Y. Su, Sci. Rep. 4, 5893 (2014).
${ }^{38}$ J. T. Robinson, J. S. Burgess, C. E. Junkermeier, S. C. Badescu, T. L. Reinecke, F. K. Perkins, M. K. Zalalutdniov, J. W. Baldwin, J. C. Culbertson, P. E. Sheehan et al., Nano Lett. 10, 3001-3005 (2010).

${ }^{39}$ S. Boopathi, T. N. Narayanan, and S. S. Kumar, Nanoscale 6, 10140 (2014).

${ }^{40}$ See supplementary material at http://dx.doi.org/10.1063/1.4922209 for details about refection loss comparison and XPS analysis. 